\title{
The temporal foundation of the principle of maximal entropy
}

\author{
Vasil Penchev< $<$ vasildinev@gmail.com \\ Bulgarian Academy of sciences: Institute of Philosophy and Sociology: \\ Dept. of Logical Systems and Models
}

\section{The hypothesis is:}

The principle of maximal entropy (further abbreviated as "MaxEnt") can be founded on the formal mechanism, in which future transforms into past by the mediation of present. This allows of MaxEnt to be investigated by the theory of quantum information.

MaxEnt can be considered as an inductive analog or generalization of "Occam's razor". It depends crucially on choice and thus on information just as all inductive methods of reasoning. The essence shared by Occam's razor and MaxEnt is for the relevant data known till now to be postulated as an enough fundament of conclusion. That axiom is the kind of choice grounding both principles. Popper's falsifiability (1935) can be discussed as a complement to them: That axiom (or axiom scheme) is always sufficient but never necessary condition of conclusion therefore postulating the choice in the base of MaxEnt. Furthermore, the abstraction axiom (or axiom scheme) relevant to set theory (e.g. the axiom scheme of specification in ZFC) involves choice analogically.

However quantum mechanics managed to demonstrate that choice and thus information should be not link to any subject in principle: Information in the form of quantum information underlies the physical world for the current of time from future to past forces this:

The subject of the paper is how that new approach to choice and information can in turn found all inductive methods and MaxEnt in particular. Indeed if choice and information can be subjectless or subject-free, then those choice and information necessarily underlying MaxEnt can be interpreted as quantum ones and consequently, subjectless or subject-free too:

The concept of quantum information introduced by quantum mechanics serves to describe uniformly future and past and thus the present as the frontier of time.

\section{Preliminary notes:}

The conception of quantum information was introduced in the theory of quantum information studying the phenomena of entanglement in quantum mechanics. The entanglement was theoretically forecast in the famous papers of Einstein, Podolsky, and Rosen (1935) and independently by Shrödinger (1935) deducing it from Hilbert space, the basic mathematical formalism of quantum mechanics. However, the former three demonstrated the forecast phenomenon as the proof of the alleged "incompleteness of quantum mechanics". John Bell (1964) deduced a sufficient condition as an experimentally verifiable criterion in order to distinguish classical from quantum correlation (entanglement). Aspect, Grangier, and Roger (1981, 1982) confirmed experimentally the existence of quantum correlations exceeding the upper limit of the possible classical correlations. The theory of quantum information has thrived since the end of the last century in the areas of quantum computer, quantum communication, and quantum cryptography. 
The fundament of quantum information is the concept of 'quantum bit', "qubit" definable as the normed superposition of any two orthogonal subspaces of complex Hilbert space as follows: 'Qubit' is: $\boldsymbol{\alpha}|\mathbf{0}\rangle+\boldsymbol{\beta}|\mathbf{1}\rangle$ where $\boldsymbol{\alpha}, \boldsymbol{\beta}$ are complex numbers such that $|\boldsymbol{\alpha}|^{2}+|\boldsymbol{\beta}|^{2}=\mathbf{1}$, and $|\mathbf{0}\rangle,|\mathbf{1}\rangle$ are any two orthonormal vectors (e.g. the orthonormal bases of any two subspaces) in any vector space (e.g. Hilbert space, Euclidean space, etc.). Thus Hilbert space underlying quantum mechanics is representable as the quantity of quantum information and any wave function, i.e. any state of any quantum system being a point in it can be seen as a value of that quantity. Consequently all physical processes turn out to be quantum-informational, and nature or the universe is a quantum computer processing quantum information.

The qubit is also isomorphic to a ball in Euclidean space, in which two points are chosen: A qubit is equivalently representable as a unit ball in Euclidean space and two points, the one chosen within the ball, and the other being the orthogonal projection on its surface, i.e. as a mapping of a unit ball onto its surface (or any other unit sphere).

Quantum information can be interpreted as the transfinite generalization of information. Its unit, a qubit, can be interpreted as a choice among an infinite set of alternatives. It generalizes the unit of classical information, a bit, which refer to a finite set of alternatives.

If one uses the notion of "length of now" defined as the period of the de Broglie (1925) wave associable to any physical item, the "length of now" of the apparatus can be visualized as a random chosen point onto the segment of the "length of now" of the measured quantum entity. Thus quantum mechanics is forced to describe uniformly the future, present and past of the investigated system. Future is represented as a coherent whole, present as a choice among infinitely many alternatives, and past as a well-ordering obtained as a result of a series of choices. The concept of quantum information unifies the future, present, and past as a measurable physical quantity. It describes the frontier of time, that "now", which transforms future into past.

The theorems about the absence of hidden variables in quantum mechanics (Neumann 1932; Kochen, Specker 1968) demonstrate that the mathematical formalism of quantum mechanics implies that no well-ordering of any coherent state might exist before measurement. However, the same coherent state is transformed into a well-ordered series of results in time after measurement. In order to be equated the state before and after measurement, the well-ordering theorem equivalent to the axiom of choice is necessary. The measurement mediating between them should be interpreted as an absolutely random choice of an element of the coherent state, for which no constructive way (equivalent to some "hidden variable") can exist in principle. The quantity of quantum information can describe uniformly the state before and after measurement (equivalent to a choice among an infinite set). Any wave function, being a given value of quantum information, "bounds" an unorderable and a well-ordered state as the quantity of qubits (i.e. the "infinite choices") necessary for the latter to be obtained from the former.

The quantity of quantum information is also the ordinal corresponding to the infinity series of finite choices. Both definitions of 'ordinal' (Cantor 1897; Neumann 1923) are applicable as the ordinals are small. The ordinal defined in Cantor - Russell (Russell, Whitehead any edition) generates a statistical ensemble while that in Neumann, a well-ordering. Both correspond one-toone to a coherent state as the one and same quantity of quantum information containing in it. 
One can investigate the following problems:

1. What is the relation between the kind of choice in quantum information and that in an inductive conclusion (particularly according to MaxEnt)?

2. Can a quantum computer calculating reversely contribute to a conclusion founded on MaxEnt?

3. What should the relation between the entropy of single data and ordered pairs, triples, etc. be according to the theory of quantum information and according to MaxEnt?

4. How does the linearity of Hilbert space limit " $n$ " in the above entropy of ordered series of "n" data?

5. How can the phenomena of non-linearity (entanglement) in quantum information be interpreted in terms of MaxEnt?

\section{References:}

Aspect, A., Grangier, R., Roger, G. (1981) "Experimental Tests of Realistic Local Theories via Bell's Theorem," Physical Review Letters 47 (7): 460-463.

Aspect, A., Grangier, R., Roger, G. (1982) "Experimental Realization of Einstein-Podolsky-RosenBohm Gedanken Experiment: A New Violation of Bell's Inequalities," Physical Review Letters 49 (2): 91-94.

Bell, J. (1964) "On the Einstein - Podolsky - Rosen paradox", Physics (New York) 1 (3): 195-200.

Broglie, L. de (1925) Recherches sur la théorie des quanta (Researches on the quantum theory), Thesis (Paris), 1924. Annales de Physique (Paris, 10-ème série) 3, 22-128.

Cantor, G. (1897) "Beitrage zur Begrundung der transfiniten Mengenlehre (Zweiter Artikel)," Mathematische Annalen 49 (2): 207-246.

Einstein, A., Podolsky, B., Rosen, N. (1935) "Can Quantum-Mechanical Description of Physical Reality Be Considered Complete?” Physical Review 47 (10): 777-780.

Kochen, S., Specker, E. (1968) "The problem of hidden variables in quantum mechanics," Journal of Mathematics and Mechanics, 17 (1): 59-87.

Neumann, J. von (1923) "Zur Einführung der trasfiniten Zahlen." Acta litterarum ac scientiarum Ragiae Universitatis Hungaricae Francisco-Josephinae, Sectio scientiarum mathematicarum 1 (4): 199208.

Neumann, J. von (1932). Mathematische Grundlagen der Quantenmechanik, Berlin: Springer, pp. 167-173 (Chapter IV.2).

Popper, K. (1935) Logik der forschung: zur erkenntnistheorie der modernen naturwissenschaft. Wien: Springer.

Schrödinger, E (1935) "Die gegenwärtige situation in der Quantenmechanik", Die Naturwissenschaften, 23 (48), 807-812; 23 (49), 823-828, 23 (50), 844-849.

Whitehead, A. N., Russell, B. (any edition) Principia Mathematica, Vol. 2(*153), Vol. 3(*251). 